\title{
Neural mediation of memory for time: Role of the hippocampus and medial prefrontal cortex
}

\author{
RAYMOND P. KESNER \\ University of Utah, Salt Lake City, Utah
}

\begin{abstract}
Within the context of the neurobiology of attribute model, memory for the temporal attribute is composed of at least three features-memory for duration, memory for succession, or temporal order, and memory for past and future time perspective within a dual-based (data and knowledge) memory system. Research aimed at testing the assumption that the hippocampus and interconnected neural circuits mediate the temporal attribute within the data-based memory system and the prefrontal cortex and interconnected neural circuits mediate the temporal attribute within the knowledge-based memory system in animals and humans is reviewed. The research indicates that (1) memory for the duration feature of the temporal attribute is mediated by the hippocampus, but not prefrontal cortex, in both animals and humans, (2) memory for the temporal order feature of the temporal attribute based on new information is subserved by both the hippocampus and the prefrontal cortex, but that based on prior knowledge or the ability to use prior knowledge is supported only by prefrontal cortex, and not the hippocampus, in both animals and humans, and (3) memory for the past (time perspective) feature of the temporal attribute is mediated by the hippocampus, whereas memory for the future (time perspective) feature of the temporal attribute is supported by the prefrontal cortex in both animals and humans. There is a clear parallel between animals and humans in terms of hippocampal and prefrontal cortex mediation of the temporal attribute, supporting the assumption of evolutionary continuity. There is support for a greater involvement of the hippocampus in comparison with the prefrontal cortex in mediating temporal attribute information within the data-based memory system. Conversely, there is support for a greater involvement of the prefrontal cortex in comparison with the hippocampus in mediating temporal attribute information within the knowledge-based memory system. Future research needs to concentrate on the development of new paradigms to measure memory for different temporal features and to uncover the critical neural circuits that subserve these temporal features.
\end{abstract}

The structure and utilization of memory is central to one's knowledge of the past, interpretation of the present, and prediction of the future. Therefore, understanding the structural and process components of memory systems at the psychological and neurobiological levels is of paramount importance. In recent years, there have been a number of attempts to divide learning and memory into multiple memory systems. Schacter and Tulving (1994) have suggested that one needs to define memory systems in terms of the kind of information to be represented, the processes associated with the operation of each system, and the neurobiological substrates, including neural structures and mechanisms that subserve each system. Furthermore, it is likely that within each system there are multiple forms or subsystems associated with each memory system, and there are likely to be multiple processes that define the operation of each system. Finally, there are probably multiple neural structures that form the overall substrate of a memory system.

Currently, the most established models of memory can be characterized as dual memory system models with an

This research was supported by NIH Grant 2ROINS20771-13. Correspondence should be addressed to R. P. Kesner, Department of Psychology, University of Utah, 502 SBS, Salt Lake City, UT 84112 (e-mail: rpkesner@behsci.utah.edu). emphasis on the hippocampus as one component of the model and a composite of other brain structures as the other component. For example, Squire (1994) has proposed that memory can be divided into a hippocampal-dependent declarative memory, which provides for conscious recollection of facts and events, and a nonhippocampal-dependent nondeclarative memory, which provides for memory without conscious access for skills, habits, priming, simple classical conditioning, and nonassociative learning. Others have used different terms to reflect the same type of distinction, including a hippocampal-dependent explicit memory versus a nonhippocampal-dependent implicit memory (Schacter, 1987) and a hippocampal-dependent declarative memory based on the representation of relationships among stimuli versus a nonhippocampal-dependent procedural memory based on the representation of a single stimulus or configuration of stimuli (Cohen \& Eichenbaum, 1993). O'Keefe and Nadel (1978) have suggested that memory is organized as a dual system based on a hippocampal-dependent representation of spatial information, labeled a locale system, and a nonhippocampaldependent representation of nonspatial information, labeled a taxon system. Olton (1983) has suggested a different dual memory system in which memory can be divided into a hippocampal-dependent working memory. defined as memory for the specific, personal, and tem- 
poral context of a situation, and a nonhippocampaldependent reference memory, defined as memory for rules and procedures (general knowledge) of specific situations. Different terms have been used to reflect the same distinction, including episodic versus semantic memory (Tulving, 1983).

However, because memory involves many neural systems in addition to the hippocampus, Kesner and DiMattia (1987) proposed a neurobiology of a dual system model organized into a data-based and knowledge-based memory system that is composed of multiple attributes or forms of memory and subserved by many different neural regions. Based on extensive research aimed at testing this dual system model, the model has been refined and updated (see Kesner, 1998, for an extensive review). In this review, I will briefly present my own comprehensive dual memory model system, followed by a detailed analysis of the temporal form or attribute of memory.

\section{NEUROBIOLOGY OF AN ATTRIBUTE MODEL}

\section{Data-Based Memory System}

In this comprehensive model, it is assumed that any specific memory is organized within a data-based memory system and a knowledge-based memory system. The data-based memory system provides for temporary representations of incoming data concerning the present, with an emphasis on facts, data, and events that are usually personal or egocentric and that occur within specific external and internal contexts. The emphasis is on the processing of new and current information. During initial learning, great emphasis is placed on the data-based memory system, which will continue to be of importance even after initial learning in situations in which unique or novel trial information needs to be remembered. The data-based memory system is composed of different independently operating forms or attributes of memory. Even though there could be many attributes, the most important attributes include space, time, response, sensory perception, and affect. In humans a language attribute is also added. The organization of these attributes within the data-based memory system can take many forms and are probably organized hierarchically and in parallel.

On the basis of a series of experiments, it can be shown that within the data-based memory system different neural structures and circuits mediate different forms or attributes of memory. The most extensive data set is based on the use of paradigms that measure the short-term or working memory process, such as matching or nonmatching to sample, delayed conditional discrimination, and continuous recognition memory of single items or lists of items. With the use of these memory paradigms, it has been shown that (1) damage to the hippocampus disrupts spatial and temporal attribute information in rats and humans and linguistic information in humans, (2) damage to the caudate affects response attributes in animals and humans, (3) damage to the amygdala impairs affect attributes in animals and humans, and (4) damage to the extrastriate visual cortex interferes with visual object information as an example of a sensory-perceptual attribute in animals and humans (Chiba, Kesner, Matsuo, \& Heilbrun, 1993; Chiba, Kesner, \& Reynolds, 1994; Heindel, Butters, \& Salmon, 1988; Hopkins, Kesner, \& Goldstein, 1995b; Horel, Pytko-Joiner, Voytko, \& Salsbury, 1987; Jackson, Kesner, \& Amann, 1994; Kesner, 1990a; Kesner, Bolland, \& Dakis, 1993; Kesner \& Williams, 1995; McDonald \& White, 1993; O'Keefe \& Nadel, 1978). Furthermore, it can be shown that these neural systems can operate independently of each other. For example, lesions of the hippocampus do not disrupt affect, response, or sensory-perceptual (odor or visual object) attributes (Chiba et al.,1993; Kesner et al., 1993; Kesner \& Williams, 1995; Leonard \& Milner, 1991; Otto \& Eichenbaum, 1992).

\section{Knowledge-Based Memory System}

The knowledge-based memory system provides for more permanent representations of previously stored information in long-term memory and can be thought of as one's general knowledge of the world. It can operate in the abstract in the absence of incoming data. The emphasis is on top-down processing. The knowledge-based memory system would tend to be of greater importance after a task has been learned, given that the situation would then be invariant and familiar. In most situations, however, one would expect a contribution of both systems with a varying proportion of involvement of one relative to the other.

The knowledge-based memory system is composed of the same set of different independently operating forms or attributes of memory. These attributes include space, time, response, sensory perception, and affect. In humans a language attribute is also added.

The organization of these attributes within the knowledge-based memory system can take many forms, and they are assumed to be organized as a set of attributedependent cognitive maps or neural nets and their interactions, which are unique for each memory. It is assumed that long-term representations within cognitive maps are more abstract and less dependent on specific features.

On the basis of a series of experiments, it can be shown that within the knowledge-based memory system, different neural structures and circuits mediate different forms or attributes of memory. The most extensive data set is based on the use of paradigms that measure the acquisition of new information, discrimination performance, executive functions, strategies, and rules to perform in a variety of tasks, including skills and the operation of a variety of long-term memory programs.

With the use of these paradigms, it has been shown that (1) damage to the posterior parietal cortex disrupts spatial attributes (DeRenzi, 1982; DiMattia \& Kesner, 1988), (2) damage to the prefrontal cortex interferes with temporal and response attributes (Fuster, 1995; Kesner \& Holbrook, 1987; Kesner, Hopkins, \& Fineman, 1994; Kolb, 1974; Passingham, 1978), (3) damage to the agran- 
ular insular and orbitofrontal cortex impairs affect attributes (Butter, 1969; Ragozzino \& Kesner, 1996), and (4) damage to the inferotemporal cortex interferes with sensory-perceptual attributes (visual object) (Fuster, 1995; Weiskrantz \& Saunders, 1984).

It is assumed that interactive patterns of independently operating attributes provide the organizational framework for the existence of each unique memory. At the neurobiological level, it is assumed that each specific brain region codes or stores the above-mentioned attributes as well as their specific interactions. Relative amounts of neural activity within each critical brain region or circuit that represents a critical attribute or a set of attributes provide for the total neural substrate associated with each unique memory representation. Thus, different neural systems are assumed to mediate specific attributes, and furthermore, each attribute is processed by different neural systems (data- and knowledge-based memory) depending on how information is processed. Finally, it is assumed that there is evolutionary continuity between animals and humans not only in terms of mnemonic function, but also in terms of brain-memory function relationships. In this paper, the emphasis will be on the temporal attribute within the context of the neurobiology of attribute model.

\section{TEMPORAL ATTRIBUTE}

Within the context of the neurobiology of attribute model, it is possible to divide the temporal attribute into different mnemonic features-namely, duration, succes- sion (or temporal order), and temporal perspective (Block, 1990). Memory for duration refers to memory for the persistence of an event or the interval between time periods or events, memory for succession refers to the sequential occurrence of events as reflected by memory for relative recency or temporal order, and memory for time perspective is based on memory for the past, present, and future and can be mediated by the use of retrospective and prospective strategies. Because time has no entity of its own, the other attributes of the model - namely, space, response, affect, sensory perception, and language-provide the content, anchors, and time markers for the memory representation of these three different temporal features. A schematic of this model is presented in Figure 1. It is assumed that the hippocampus and interconnected neural circuits in rats and humans mediate the temporal attribute within the data-based memory system and that the medial prefrontal cortex (anterior cingulate) and interconnected neural circuits in the rat and the dorsolateral prefrontal cortex and interconnected neural circuits in humans and monkeys mediate the temporal attribute within the knowledge-based memory system. It should be noted that important anatomical and functional links exist between the hippocampus and prefrontal cortex that are likely to aid in the representation and processing of temporal information, but that also will provide for difficulties in dissociating the contributions of the hippocampus and prefrontal cortex in supporting temporal information. In the rat, the neural connections form a circuit starting with a direct hippocampal CAl/subiculum pro-

\section{T I ME}
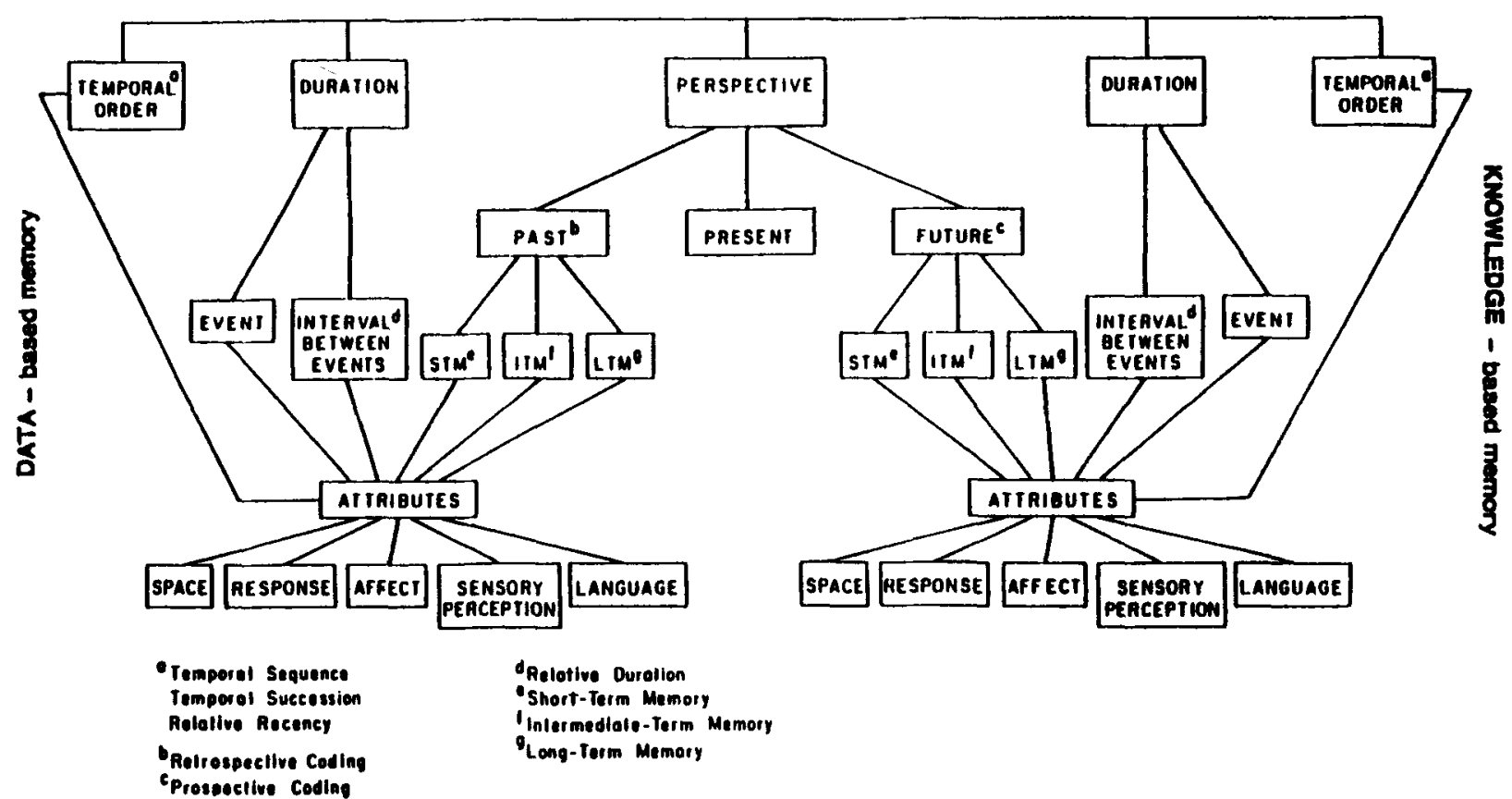

Figure 1. A schematic of the organization of temporal attribute features within the data-based and knowledge-based memory systems. 
jection to the medial prefrontal cortex, especially the infralimbic/prelimbic cortex (Ferino, Thierry, \& Glowinski, 1987; Swanson, 1981). Interestingly, one can elicit long-term potentiation (LTP) by stimulation of the CA1 hippocampal region and recording from the infralimbic cortex (Laroche, Jay, \& Thierry, 1990). The medial prefrontal cortex is directly connected to the entorhinal cortex, which, in turn, is directly connected to the $\mathrm{CAl}$ and subiculum, thereby completing the circuit (Witter, Groenewegen, Lopes Da Silva, \& Lohman, 1989). A similar circuit exists in the monkey in that the hippocampus connects to the subiculum, which, in turn, projects directly to the dorsolateral prefrontal cortex. The dorsolateral prefrontal cortex projects to the presubiculum, entorhinal cortex, and parahippocampal cortices, which, in turn, project to the hippocampus (GoldmanRakic, Selemon, \& Schwartz, 1984; Uylings \& van Eden, 1990; Witter et al., 1989).

The purpose of this review is to determine whether some empirical support exists for the above-mentioned assumptions. The major questions to be asked are whether each neural system mediates all three of the temporal features associated with the temporal attribute, whether it is possible to dissociate the temporal attribute functions of the hippocampus and prefrontal cortex, and whether there are parallels between animal and humans in terms of the neural basis of memory for the temporal attribute.

\section{Memory for Duration-Animals}

In this section, I will concentrate on memory for duration rather than the processing of time and time perception as measured by time estimation and time-scale invariance (for a more recent review, see Gibbon, Malapani, Dale, \& Gallistel, 1997). Previous research has indicated that fimbria-fornix-lesioned, but not frontal-cortexlesioned, rats are impaired in remembering the duration of a stimulus across a short delay interval, even though there is only a small change in estimating the passage of time (Meck, Church, \& Olton, 1984; Olton, 1986; Olton, Wenk, Church, \& Meck, 1988). In an attempt to replicate these results with a different paradigm, rats were trained on a short-term memory for duration task using a delayed symbolic conditional discrimination procedure (Jackson et al., 1994). It had previously been shown that rats can acquire high proficiency in short-term memory for duration information (Santi, Weise, \& Kuiper, 1995). In the Jackson et al. experiment, the rats had to learn that a black rectangle stimulus that was visible for $2 \mathrm{sec}$ would result in a positive (go) reinforcement for one object (a ball) and no reinforcement (no go) for a different object (a bottle). However, if the black rectangle stimulus was visible for $8 \mathrm{sec}$, then there would be no reinforcement for the ball (no go), but there would be a reinforcement for the bottle (go). After rats learned to respond differentially in terms of latency to approach the object, they received large (dorsal and ventral) lesions of the hippocampus, lesions of the medial prefrontal cortex (anterior cingulate), or lesions of the cortex dorsal to the dorsal hippocampus. Following recovery from surgery they were retested. The results show that in contrast to cortical control and medial prefrontal cortex lesions, major impairments followed hippocampal lesions, as indicated by smaller and statistically nonsignificant latency differences between positive and negative trials on postsurgery tests. In order to ensure that the deficits observed with hippocampal lesions were not due to a discrimination problem, new rats were trained in an object (black rectangle) duration discrimination task. In this situation, the rats were reinforced for either a 2- or a 10-sec exposure (duration) of the black rectangle. The stimulus was presented and remained visible for either 2 or $10 \mathrm{sec}$, following which the door was raised and latency to move the stimulus was measured. Half of the animals in each group received a piece of Froot Loop on trials with a short stimulus duration, and the other half was reinforced on those trials with a long stimulus duration. After rats learned to respond differentially in terms of latency to approach the object, they received large (dorsal and ventral) lesions of the hippocampus, as well as medial prefrontal cortex lesions for comparison purposes, or lesions of cortex dorsal to the dorsal hippocampus. Following recovery from surgery, the rats were retested. The results indicate that after hippocampal lesions, there was an initial deficit followed by complete recovery. There were no significant changes for the medial-prefrontal- or controllesioned animals.

Thus, the hippocampus mediates memory for duration, but does not mediate duration discrimination. The data are consistent with those from previous research indicating that fimbria-fornix-lesioned, but not frontalcortex-lesioned, rats are impaired in remembering the duration of a stimulus across a short delay interval, even though there is only a small change in estimating the passage of time (Meck et al., 1984; Olton, 1986; Olton et al., 1988). Furthermore, it has been suggested that trace conditioning requires memory for the duration of the conditioned stimulus. Thus, it is of importance to note that rabbits with hippocampal lesions are impaired in acquisition (consolidation) of trace, but not delayed, eye-blink conditioning (Moyer, Deyo, \& Disterhoft, 1990). In rats, it appears that the hippocampus is actively involved in representing in short-term memory temporal attribute information that is perhaps based on the use of markers for the beginning and end of the presence (duration) of a stimulus (object). It should be noted that the hippocampus is not directly involved in representing memory information concerning specific objects (Kesner et al., 1993; Mumby, Wood, \& Pinel, 1992). The medial prefrontal cortex does not appear to play a role in either memory for duration or memory for duration discrimination. It is possible that other neural regions within the prefrontal cortex could mediate memory for duration, but the anterior cingulate does not appear to be critical.

\section{Memory for Duration-Humans}

To what extent can one generalize from hippocampal and anterior cingulate function in rats to humans with respect to memory representation of duration as one feature 
of temporal attribute information? Since there are no data in the literature that address this question, a number of experiments were conducted using humans that were exposed to hypoxia due to a variety of causes, but primarily due to carbon monoxide poisoning (Hopkins \& Kesner, 1994, 1995). These subjects had a profound anterograde amnesia and, according to MRI data, had bilateral damage to the hippocampus, but no detectable damage to entorhinal cortex, parahippocampal gyrus, or temporal cortex. They also showed no signs of prefrontal cortex dysfunction, with normal performance on tests of fluency and Wisconsin card sorting. In addition, patients with damage to the prefrontal cortex were used. The hypoxic and prefrontal-cortex-damaged subjects and agematched controls were tested for short-term memory for duration of a visual object. Subjects were presented with a single object (square, circle, etc.) on a computer screen for a duration of 1 or $3 \mathrm{sec}$. They were instructed to remember the duration of presentation of the object. After a delay of $1,4,8,12,16$, or $20 \mathrm{sec}$, the same object appeared for the same or a different duration. The subjects were asked to indicate whether the duration was the same or different from the duration shown in the study phase. The results indicate that the hypoxic subjects were impaired relative to control and prefrontal-cortex-damaged subjects in short-term memory for duration for all delays. There were no deficits for the prefrontal-cortexdamaged subjects. In order to determine whether the deficits may have been due to impaired memory for the objects per se, a control task was administered to the same subjects. They were presented with a single object for a duration of 1 or $3 \mathrm{sec}$ and were asked to remember the object. After a delay of $1,4,8,12,16$, or $20 \mathrm{sec}$, either the identical or a different object appeared on the screen. The subjects were asked if it was the same or a different object. The results indicate that there were no significant differences between the hypoxic, prefrontal-cortex, and control subjects. The impairment could not have been due to an inability to estimate time accurately, because in an additional experiment with objects, the subjects were asked to estimate the time elapsed before each of the 1-, 4-, 8-, $12-, 16-$, and $20-\mathrm{sec}$ delay intervals. The results indicate that for hypoxic subjects, time estimates were accurate up to $8 \mathrm{sec}$, followed by some underestimation with longer delays, so that short-term memory for the duration of 1 or 3-sec stimulus exposure could not have been due to difficulty in estimating time. The process of estimating time may not require active participation of short-term memory and may, therefore, be independent of shortterm memory for the duration of exposure of a stimulus. Similarly, for the prefrontal-cortex-damaged subjects, time estimates were accurate up to $8 \mathrm{sec}$, followed by a slight underestimation with longer delays.

The data are consistent with previous findings indicating that humans with hypoxia resulting in bilateral hippocampal damage are impaired in acquisition (consolidation) of trace but not delayed eye-blink conditioning (Disterhoft, Carrillo, Hopkins, Gabrieli, \& Kesner,
1996). Thus, the results suggest that like rodents, humans with hippocampal damage have difficulty in representing short-term memory for duration of an object, but not shortterm memory for a single object. In contrast, both rodents and humans with prefrontal cortex damage do not appear to have any difficulty in short-term memory for duration of an object, suggesting a dissociation between hippocampal and prefrontal cortex representation of duration information.

\section{Memory for Temporal Order-Animals}

In order to test whether the hippocampus or medial prefrontal cortex subserves memory for temporal order, rats were tested for order memory for a list of items (places on a maze) (Kesner \& Holbrook, 1987; Kesner \& Novak, 1982). Rats were trained on an eight-arm radial maze for Froot Loop reinforcement. After training, each animal was allowed to visit eight arms on each trial (one per day) in an order that was randomly selected for that trial (study phase). The sequencing of the eight arms was accomplished by sequentially opening Plexiglas doors (one at a time) located at the entrance of each arm. Immediately after the animal had received reinforcement from the last of the eight arms, the test phase began. During the test phase for order memory, either the first and second, fourth and fifth, or seventh and eighth doors that occurred in the sequence were opened. The rule to be learned, leading to an additional reinforcement, was to choose the arm that occurred earlier in the sequence. There were 24 tests with only 1 test per trial.

Following training, animals performed better than chance for the first- (primacy) and last- (recency) order positions. The animals then received medial prefrontal cortex or dorsal hippocampal lesions. After recovery from surgery, animals were given an additional 24 tests. Results indicate that medial prefrontal-cortex-lesioned animals had an order memory deficit for all spatial locations. In additional tests, it was shown that this order deficit appeared even when the animals were allowed to self-order the spatial locations during the study phase, were presented with a fixed sequence of four spatial locations, or when the list length was only two spatial locations (Kesner \& Holbrook, 1987). In contrast, dorsalhippocampal-lesioned rats were impaired only for the first and middle choice orders, but they performed without difficulty on the last choice order; that is, they showed a primacy deficit, but an intact recency effect. The normal recency effect could have been due to small lesions of the hippocampus. Larger lesions (dorsal and ventral) of the hippocampus also impair the recency effect (unpublished observations). These data suggest that both neural regions play a role in memory for temporal order.

The possibility exists, however, that order memory was impaired in the Kesner and Holbrook (1987) and Kesner and Novak (1982) experiments, because only temporally adjacent items were selected. This is especially a problem because Estes (1986) has summarized data in humans demonstrating that order or sequential information is re- 
membered better when more items (lag) occur between any two items to be tested. Thus, an experiment was designed to test order memory for items that were further apart temporally in the to-be-remembered sequence (Chiba, Johnson, \& Kesner, 1992; Chiba, Kesner, \& Reynolds, 1994). In this experiment, rats are required to remember an event (e.g., spatial location, visual object) dependent on the temporal order of the occurrence of events. More specifically, on an eight-arm maze during the study phase of each trial, rats were allowed to visit each of the eight arms once in an order that had been randomly selected for that trial. The test phase required the rats to choose which of two arms occurred earlier in the sequence of arms visited during the study phase. The arms selected as test arms varied according to temporal lag or distance $(0-6)$ or the number of arms that occurred between the two test arms in the study phase. After the rats reached a criterion of $75 \%$ or better performance on all the distances but zero, they received large (dorsal and ventral), small (dorsal) hippocampus, medial prefrontal cortex, or cortical control lesions dorsal to the dorsal hippocampus. Following recovery from surgery, the rats were retested. The results indicate that for postsurgery tests, the control rats performed at chance at a temporal distance of zero, but their performance was excellent for the remaining temporal distances. In contrast, rats with dorsal hippocampal lesions did not perform well for temporal distances of 0 and 2 on postsurgery tests, but performance was excellent for the longest temporal distances of 4 and 6. Furthermore, on postsurgery tests rats with large (dorsal plus ventral) hippocampal and medial prefrontal cortex lesions had a marked deficit for all temporal distances, with a slight improvement for the longest temporal distance. In this task, it is necessary to separate one event from another. Hippocampal-lesioned or medial-prefrontalcortex-lesioned rats cannot separate events across time, perhaps because of an inability to inhibit interference that is likely to accompany sequentially occurring events. The resultant increase in temporal interference impairs the rat's ability to remember the order of specific events. It appears that the larger the damage to the hippocampus, the greater the temporal interference.

In the previous experiment, the selected sequence to be remembered varied on each trial, emphasizing the importance of new information, which is likely to be processed by the data-based memory system. An alternative procedure is based on the presentation of a single fixed or constant sequence to be remembered. After the temporal order of this sequence is learned, there is a high likelihood that the knowledge-based system rather than the data-based system would mediate memory for this constant order. On the basis of the hypothesis that the medial prefrontal cortex rather than the hippocampus mediates temporal order within the knowledge-based memory system, it should be possible to determine whether the medial prefrontal cortex plays a more important role than the hippocampus in the constant temporal distance task. To test this idea, rats were presented with a constant sequence of eight spatial locations, followed by tempora distance tests. The results indicate that relative to con. trols, small and large hippocampal lesions do not resul in any significant deficits, but medial prefrontal cortex lesions do produce a significant impairment (Chibs et al., 1992; Chiba et al., 1994). Thus, these data suppor the importance of medial prefrontal cortex for maintaining well-learned temporal sequences in long-term memory It should be noted that the events do not have to be basec on only spatial location information. Similar deficits have been observed with lists of visual objects in rats (unpublished observations).

In general, these data are consistent with previous descriptions of deficits following medial prefrontal cortex or hippocampal lesions in tasks in which rats emit a specific sequence of behavioral responses requiring temporal organization (Barker, 1967; Slotnick, 1967; Stamm. 1955). In monkeys, dorsolateral prefrontal cortex lesions result in deficits in temporal ordering of events comparable to what has been described in rats. This temporal ordering deficit is evidenced by deficits in self-ordering of a sequence of responses (Petrides \& Milner, 1982). In another experiment, by Pribram and Tubbs (1967), monkeys with frontal cortex lesions could not perform a 5-sec right-left delayed alternation task (order deficit). but with a 15 -sec delay between a right-left couplet of responses, no deficit was found. In the latter condition. the animal could chunk, or categorize, each couplet as an item of information and did not need to remember the order of a right-left response. In a more recent study, Funahashi, Inoue, and Kubota (1997) showed that neurons within the prefrontal cortex (periprincipal and superior arcuate areas) of primates participated in retaining the temporal order of stimulus presentation during the delay period prior to a test for temporal order memory. On the basis of the above-mentioned findings, it has been suggested that the prefrontal cortex is primarily involved in temporal structuring of information in short-term memory (Fuster, 1980).

A somewhat different function based on temporal order relates to memory for frequency of occurrence of events. In order to test the role of the hippocampus and medial prefrontal cortex in memory for frequency, rats were tested on an eight-arm radial maze (Kesner, 1990b). On each trial (one per day), each animal was allowed to visit four arms to receive reinforcement in an order that had been randomly selected for that trial. One of the arms was repeated with a lag of one, two, or three arms in between the repetition. The sequencing of the four arms and the repetition of one of the arms was accomplished by sequentially opening Plexiglas doors to each arm (one at a time). This constituted the study phase. Within $20 \mathrm{sec}$ after entry into the last (fifth) arm of the study phase, the door in front of the repeated arm and the door in front of a nonrepeated arm were opened simultaneously. This constituted the test phase. The rule to be learned, leading to an additional Froot Loop reinforcement, was to choose the arm that had been repeated in the study phase sequence. 
After training, and on the basis of at least eight observations per lag, animals showed excellent memory for the repetition, with a lag of three arms between repetitions, but performed poorly for a lag of one or two arms. The effect was not due to the variable number of spatial locations that occurred between the repeated spatial locations and the test, because analysis of only the repeated spatial locations that occurred at the end or the beginning of the list revealed the same repetition lag effect. This repetition lag effect (i.e., better retention with more items between a repetition) has also been reported for humans (Madigan, 1969). The rats then received medial prefrontal cortex or hippocampus lesions or served as sham-operated controls. After recovery from surgery, animals were given an additional 24 tests, with 8 tests for each lag condition. Sham-operated animals showed no significant changes in performance. In contrast, animals with medial prefrontal cortex or hippocampus lesions displayed a deficit for all lag conditions. Thus, both lesions disrupted the repetition lag effect, further supporting a role for the hippocampus and medial prefrontal cortex in mediating the temporal order feature of the temporal attribute.

\section{Memory for Temporal Order-Humans}

Previous research with frontal lobe-damaged patients indicate that these patients cannot (1) remember the order in which information has been experienced, (2) plan and create a complex set of motor movements, or (3) program a temporally ordered set of activities. For example, it has been shown that frontal cortex-damaged patients cannot discriminate the more from the less recent, even though they can remember that certain words or pictures have been presented (Lewinsohn, Zieler, Libet, Eyeberg, \& Nielson, 1972; Milner, 1971). In addition, frontal cortexdamaged patients were impaired in a short-term memory task in which two stimuli had to be remembered for a 60 sec time interval (paired-comparison task) (Milner, 1964). This task requires short-term temporal memory for two events, implying that frontal cortex-damaged patients cannot remember the order of stimulus presentation. In another experiment, frontal cortex-damaged patients were impaired in their ability to self-order a sequence of stimuli presented one at a time (Petrides \& Milner, 1982). Additional evidence comes from a study in which frontal lobedamaged patients were very poor at copying a series of facial movements. These patients had difficulty in ordering the various components of the sequence. The components were remembered correctly, but were expressed in an incorrect order (Kolb \& Milner, 1981).

Previous research indicates that amnesic patients also have difficulty in processing temporal order information (Hirst \& Volpe, 1982; Huppert \& Piercey, 1976; Squire, Nadel, \& Slater, 1981). Only one study, Sagar, Gabrieli, Sullivan, and Corkin (1990), found no deficit for patient H.M., who had a bilateral temporal lobectomy. However,
Sagar et al. used a different testing procedure for assessing temporal order memory. Reanalyzing Sagar et al.'s data using a temporal distance measure, as described below, resulted in the same pattern of results. Even though the tasks used in previous studies are not analogous to those that have been used in rat studies, is it possible to generalize from hippocampal and medial prefrontal cortex (anterior cingulate) function in rats to humans with respect to memory representation of temporal succession as one feature of temporal attribute information? In order to answer this question, tasks were selected that were more analogous to those of previous research with rats, namely short-term order memory for a six-item list of Xs that appear in specific locations. Furthermore, to compare the right and left sides in humans, a comparable task using words was also used. In this study, patients with right or left temporal lobe resection; hypoxics with damage to the hippocampus; bilateral, left, and right prefrontal cortex; epileptic controls; and age-matched controls were tested for order memory. More specifically, for the spatial task during the study phase, a series of random (novel) sequences of six Xs appeared on the screen for a period of $5 \mathrm{sec}$ each. Subjects were instructed to pay attention to the locations of the Xs as well as to the order in which they occurred. In the test phase, subjects were presented with two Xs and were asked to determine which one had occurred earlier in the study phase. For the verbal task during the study phase, a series of random (novel) sequences of six words appeared on the screen for a period of $5 \mathrm{sec}$ each. Subjects were instructed to pay attention to the words as well as to the order in which they occurred. In the test phase, subjects were presented with words and were asked to determine which one had occurred earlier in the study phase. The procedures used for the order memory tests were first developed for the assessment of list learning for spatial location information in rats and were then adapted for use with brain-damaged patients. Because in rats only one test was given for each serial list, the same procedure was adopted for humans. This procedure has the advantage of minimizing response interference. Because the set size is only six items, normal subjects perform well on both order memory tests. The results indicate that relative to age-matched controls for spatial location temporal order memory, the right temporal lobe-resected patients which included hippocampal damage, right prefrontal cortexdamaged patients, and hypoxic patients were markedly impaired. For word temporal order memory relative to agematched controls, the left temporal lobe-resected patients which included hippocampal damage, left and bilateral prefrontal cortex-damaged patients, and hypoxic patients which were markedly impaired. Similar results were obtained for temporal order for abstract pictures and motor responses. Also, for frontal cortex-damaged subjects, there were order memory deficits but no deficits for memory for items per se; for hippocampal-damaged subjects, however, there were deficits not only for order 
but also for item information (Hopkins, Kesner, \& Goldstein, 1995a; Kesner, 1990a; Kesner \& Holbrook, 1987; Kesner et al., 1994).

On the basis of the demonstration that in humans order or sequential information is remembered better when more items (lag) occur between any two items to be tested, an additional experiment was designed to test order or distance memory for items that are further apart temporally in the to-be-remembered sequence. Patients with right temporal lobe resection, hypoxics with damage to the hippocampus, right prefrontal cortex, and controls were presented with a list of eight spatial locations (Xs) on a grid on a Macintosh computer and tested for memory for temporal distances (Chiba, Kesner, Matsuo, Heilbrun, \& Plumb, 1998; Hopkins et al., 1995b).

In this task, during the study phase a series of random (novel) sequences of eight Xs appeared on the screen for a period of $5 \mathrm{sec}$ each. Subjects were instructed to pay attention to the locations of the Xs as well as to the order in which they occurred. In the test phase, subjects were presented with two Xs and were asked to determine which one had occurred earlier in the study phase. Unlimited time was allowed for the subjects to make their choices. Temporal distances of $0,2,4$, and 6 were assessed with eight observations for each distance for each type of task. Temporal distance is determined on the basis of the number of items in the study phase that occurs between the two test items. For each study phase, four tests were given, one for each temporal distance. The results indicate that relative to controls, the hypoxics and the righttemporal-lobe-resected subjects showed an impairment for all temporal distances, but with some improvement for the longest distances, whereas the right-prefrontalcortex-damaged patients showed a deficit for all temporal distances. These results are similar to what was found in rats and suggest that patients with right or bilateral hippocampal dysfunction have difficulty in encoding or remembering temporal contexts for spatial information, perhaps due to increased interference in the temporal resolution of specific spatial locations. In the previous experiment, the selected sequence to be remembered varied on each trial, emphasizing the importance of new information, which is likely to be processed by the databased memory system. An alternative procedure is based on the presentation of meaningful geometric patterns, to emphasize the contribution of the knowledge-based memory system. On the basis of the hypothesis that the medial prefrontal cortex rather than the hippocampus mediates temporal order within the knowledge-based memory system, it should be possible to determine whether the medial prefrontal cortex plays a more important role than the hippocampus in a structured spatial temporal distance task. To test this idea further, the same subjects were given sequences in which the Xs appeared in a meaningful geometric pattern. An example of a meaningful and familiar pattern would be the presentation of the locations in the pattern of a large $X$. The first
$X$ would be presented in the top-left corner of the grid and then in each subsequent square down the diagonal, ending in the bottom-right corner. The next $\mathrm{X}$ would be presented in the bottom-left corner of the grid and in each subsequent square up the diagonal, ending in the topright corner. The results indicate that relative to controls, there were no deficits for the right-temporal-lobe-resected patients or the hypoxic patients, whereas there was a profound deficit for the right-frontal-cortex-damaged subjects for all but the longest temporal distance.

In general, the above-mentioned data support the idea that the hippocampus and prefrontal cortex in rats and humans mediate new temporal order information, but when the information is well known or a structured rule can be applied, only the prefrontal cortex in rats and humans continues to mediate temporal order information. These data suggest that whenever knowledge-based information can be used to remember temporal order of specific sequences of events, there is a greater involvement of the prefrontal cortex in comparison with the hippocampus. It is also important to point to significant parallels in mediating temporal order information between animals and humans.

Since temporal organization is required for frequency memory (repetition), it is not surprising that patients with prefrontal cortex damage, and to some extent, temporallobe-resected patients with hippocampal damage, are impaired in judgment for frequency of occurrence of abstract designs within a list (Milner, Petrides, \& Smith, 1985). In a more recent study, Jurado, Junque, Pujol, Oliver, and Vendrell (1997) showed that frontal-lobedamaged patients had a similar impairment in estimating the frequency of occurrence of words within a list, yet item recognition for those same words remained intact. It has been suggested that frequency judgments require a search through memory for temporal information (Hintzman, Grandy, \& Gold, 1981; Hintzman, Nozawa, \& Irmscher, 1982; Milner et al., 1985); that is, one needs to search one's memory for each specific instance of a specific item. In summary, it appears that the prefrontal cortex in humans and the medial prefrontal cortex in the rat mediate memory for frequency, further supporting the importance of prefrontal cortex and hippocampus in mediating temporal order information.

\section{Time Perspective: Memory for the Past and Future-Animals, Humans}

It has been suggested that the hippocampus mediates memory for past events within a relatively short time frame (short-term, intermediate-term, or working memory) (Kesner, 1990a; Olton, 1983). Thus, as a consequence of damage to the hippocampus, one would expect severe difficulty in remembering relatively new spatiotemporal information, which would, in turn, create a problem in using memory for the future that is based on memories necessary to plan or anticipate future events. In contrast, it has been suggested that the prefrontal cortex mediates memory 
for future events, given that memory for the past within either a short or long time frame is not based on temporal order.

In order to test these ideas in rats, sham-operated rats or animals with medial prefrontal cortex or hippocampus lesions were tested in a task that provided an opportunity for rats to utilize retrospective (past as time perspective) and prospective (future as time perspective) memory codes, while remembering items (spatial locations) within short or long lists (Kametani \& Kesner, 1989; Kesner, 1989). More specifically, on any one trial a rat was presented with $2,4,6,8$, or 10 items (spatial locations) on a 12 -arm radial maze followed 15 min later by two win-shift tests constituting a choice between a place previously visited and a novel place. Each animal was given a total of 20 trials with eight tests for each point of interpolation or each list length $(2,4,6,8$, or 10$)$. During learning, rats showed an increase in errors as the number of places to be remembered was increased from 2 to 6 to 8 , reflecting the use of a retrospective memory code (the ability to remember the spatial locations previously visited). These rats also showed a decrease in errors as the number of places was increased from 8 to 10 , reflecting the use of a prospective memory code (the ability to anticipate the spatial locations that have not yet been visited). Results indicate that sham-operated animals displayed an increase in errors as a function of point of interpolation or set size (2-8 items) followed by a decrease in errors with a set size of 10 items, suggesting the use of both retrospective and prospective memory codes. In contrast, animals with medial prefrontal cortex lesions made few errors for short list lengths, but many errors for the longer list lengths, reflecting an inability to shift from a retrospective to a prospective memory code, whereas animals with hippocampus lesions made errors across all list lengths, reflecting a deficit in utilizing both retrospective and prospective strategies. It is likely that the hippocampuslesioned rats were not able to use a prospective strategy, because they were not able to remember spatial information needed to initiate a prospective strategy.

Additional support for the idea that the hippocampus mediates the past comes from the findings that posttrial disruption of normal hippocampal function with, for example, electrical brain stimulation, results in timedependent memory impairments (Kesner \& Wilburn, 1974). These effects reveal that the hippocampus is involved in short-term consolidation processes, because the gradients are usually short-within minutes to a few hours. In a more recent study (Kim, Clark, \& Thompson, 1995 ), it was shown that trace eye-blink conditioning is impaired when hippocampal lesions are made 1 day, but not 30 days, after acquisition, suggesting that trace conditioning as a measure of duration information reveals the involvement of the hippocampus in representing the past via short-term consolidation processes. Long-term temporally graded functions have also been observed for previously learned spatial discriminations prior to surgery in rats and mice, but these long-term gradients (2-4 weeks) have been observed primarily following entorhinal cortex rather than hippocampal lesions (Cho, Beracochea, \& Jaffard, 1993; Cho \& Kesner, 1996; Cho, Kesner, \& Brodale, 1995). A long-term gradient following hippocampal lesions has been reported following contextual fear conditioning (Kim \& Fanselow, 1992), but recent results have cast doubt on whether these gradients can be reliably measured (Maren, Aharonov, \& Fanselow, 1996; Weisend, Astur, \& Sutherland, 1996). Thus, it is possible that short-term consolidation gradients derive from hippocampal dysfunction, whereas entorhinal cortex dysfunction is necessary to produce longterm retrograde amnesia consolidation gradients. Very long term memory losses often, observed in patients with damage to the hippocampus and interconnected neural circuitry, tend to result in flat retrograde amnesia gradients, suggesting a role for the hippocampus in retrieving very long term information (for a review, see Nadel \& Moscovitch, 1997). Whether the hippocampus promotes the transfer of information to the knowledge-based system, promotes the consolidation of information already processed in the knowledge-based system, or is intimately involved in retrieval of past information, still needs to be resolved.

The results of the Kametani and Kesner (1989) study indicate that the medial prefrontal cortex mediates prospective memories in an item recognition task that utilizes spatiotemporal information, suggesting an important role for the medial prefrontal cortex in mediating a future time perspective within the knowledge-based memory system. Fuster (1985) has also suggested that dorsolateral prefrontal cortex of monkeys and humans mediates prospective functions of temporal ordering of information. Support for this possible prospective function comes from the finding that some cells in the dorsolateral frontal cortex of monkeys show a gradual increase in their firing rate during the delay, in apparent anticipation of the test phase of a delayed matching-tosample, delayed response, or delayed alternation task (Fuster, Bauer, \& Jervey, 1982; Kojima, Matsumuru, \& Kubota, 1981; Niki, 1974a, 1974b). Furthermore, Shallice (1982) and Owen, Downes, Sahakian, Polkey, and Robbins (1990) have shown that left-frontal-lobe-damaged patients have difficulty solving the Tower of London problem. This problem requires temporal ordering of simple moves and thus requires planning and the utilization of prospective codes. Also, during learning of the Tower of London task and on the basis of SPECT or PET scans, increased cerebral blood flow was measured in the left dorsolateral prefrontal cortex, but it should be noted that other cortical areas are also activated, including parietal cortex and other frontal cortical areas (Baker et al., 1996; Morris, Ahmed, Syed, \& Toone, 1993). Finally, in another experiment, it was shown that frontalcortex-damaged subjects were impaired in their ability to self-order a sequence of stimuli presented one at a time. It is assumed that to perform well in this task, the subject has to initiate a plan to follow and has to initiate the order 
of responding (Petrides \& Milner, 1982). Finally, it should be noted that there is some evidence that the prefrontal cortex also plays a role in representing the past in that damage to the lateroventral prefrontal cortex, in particular, produces a long-term retrograde amnesia gradient, which has been interpreted to reflect a loss or an impairment in the use of strategic retrieval processes (Markowitsch, 1995; Nadel \& Moscovitch, 1997).

\section{REFERENCES}

Baker, S. C., Rogers, R. D., OWen, A. M., Frith, C. D., Dolan, R. J., Frackowiak, R. S. J., \& RobBins, T. W. (1996). Neural systems engaged by planning: A PET study of the Tower of London task. Neuropsychologia, 34, 515-526.

BARKER, D. J. (1967). Alterations in sequential behavior of rats following ablation of midline limbic cortex. Journal of Comparative \& Physiological Psychology, 3, 453-604.

BLock, R. A. (1990). Models of psychological time. In R. A. Block (Ed.), Cognitive models of psychological time (pp. 1-36). Hillsdale, NJ: Erlbaum

BUtTER, C. M. (1969). Perseveration in extinction and in discrimination reversal tasks following selective frontal ablations in Macaca mulatta. Physiology \& Behavior, 4, 163-171.

Chiba, A. A., Johnson, D. L., \& Kesner, R. P. (1992). The effects of lesions of the dorsal hippocampus or the ventral hippocampus on performance of a spatial location order recognition task. Society for $\mathrm{Neu}$ roscience Abstracts, 18, 1422.

Chiba, A. A., Kesner, R. P., Matsuo, F., \& Hellbrun, M. P. (1993). A dissociation between affect and recognition following unilateral temporal lobectomy including the amygdala. Society for Neuroscience Abstracts, 19, 792.

Chiba, A. A., Kesner, R. P., Matsuo, F., Heilbrun, M. P., \& Plumb, S. (1998). A double dissociation between the right and left hippocampus in processing the temporal order of spatial and verbal information. Manuscript submitted for publication.

Chiba, A. A., Kesner, R. P., \& Reynolds, A. M. (1994). Memory for spatial location as a function of temporal lag in rats: Role of hippocampus and medial prefrontal cortex. Behavioral \& Neural Biology, 61, 123-131

Cho, Y. H., Beracochea, D., \& Jaffard, R. (1993). Extended temporal gradient for retrograde and anterograde amnesia produced by ibotenate entorhinal cortex lesions in mice. Journal of Neuroscience. 13, 1759-1766.

Cho, Y. H., \& KeSNer, R. P. (1996). Involvement of entorhinal cortex or parietal cortex in long-term spatial discrimination memory in rats: Retrograde amnesia. Behavioral Neuroscience, 110, 436-442.

Cho, Y. H., Kesner, R. P., \& Brodale, S. (1995). Retrograde and anterograde amnesia for spatial discrimination in rats: Role of hippocampus, entorhinal cortex, and parietal cortex. Psychobiology, 23, 185-194.

Cohen, N. J., \& Eichenbaum, H. B. (1993). Memory, amnesia, and hippocampal function. Cambridge, MA: MIT Press.

DERENZI, E. (1982). Disorders of space exploration and cognition. New York: Wiley.

DiMattia, B. V., \& Kesner, R. P. (1988). Spatial cognitive maps: Differential role of parietal cortex and hippocampal formation. Behavioral Neuroscience, 102, 471-480.

Disterhoft, J. F., Carrillo, M. C., Hopkins, R. O., Gabrieli, J. D. E., \& KESNER, R. P. (1996). Impaired trace eyeblink conditioning in severe medial temporal lobe amnesics. Society for Neuroscience $A b$ stracts, 22, 1866.

EStes, W. K. (1986). Memory for temporal information. In J. A. Michon \& J. L. Jackson (Eds.), Time, mind and hehavior (pp. 151-168). New York: Springer-Verlag.

Ferino, F., Thierry, A. M., \& Glowinski, J. (1987). Anatomical and electrophysiological evidence for a direct projection from Ammon's horn to the medial prefrontal cortex in the rat. Experimental Brain Research, 65, 421-426.
Funahashi, S., Inoue, M., \& Kubota, K. (1997). Delay-period activity in the primate prefrontal cortex encoding multiple spatial positions and their order of presentation. Behavioural Brain Research, 84, 203-223.

FusTER, J. M. ( 1980). The prefrontal cortex: Anatomy, physiology, and neuropsychology of the frontal lobe. New York: Raven.

FUSTER, J. M. (1985). The prefrontal cortex, mediator of cross-temporal contingencies. Human Neurobiology, 4, 169-179.

Fuster, J. M. (1995). Memory in the cerebral cortex: An empirical approach to neural networks in the human and nonhuman primate. Cambridge, MA: MIT Press.

Fuster, J. M., Bauer, R. H., \& Jervey, J. P. (1982). Cellular discharge in the dorsolateral prefrontal cortex of the monkey in cognitive tasks. Experimental Neurology, 77, 679-694.

Gibbon, J., Malapani, C., Dale, C. L., \& Gallistel, C. R. (1997). Toward a neurobiology of temporal cognition: Advances and challenges. Current Opinion in Neurobiology, 7, 170-184.

Goldman-Rakic, P. S., Selemon, L. D., \& Schwartz, M. L. (1984). Dual pathways connecting the dorsolateral prefrontal cortex with the hippocampal formation and parahippocampal cortex in the rhesus monkey. Neuroscience, 12, 719-743.

Heindel, W., Butters, N., \& Salmon, D. (1988). Impaired learning of a motor skill in patients with Huntington's disease. Behavioral Neuroscience, 102, 141-147.

Hintzman, D. L., Grandy, C. A., \& Gold, E. (1981). Memory for frequency: A comparison of two multiple trace theories. Journal of Experimental Psychology: Human Learning \& Memory, 7, 231-240.

Hintzman, D. L., Nozawa, G., \& Irmscher, M. (1982). Frequency as a nonpropositional attribute of memory. Journal of Verbal Learning \& Verbal Behavior, 21, 127-141.

HiRST, W., \& VOLPE, B. (1982). Temporal order judgments with amnesia. Brain \& Language, 1, 294-306.

HoPKINS, R. O., \& KESNER, R. P. (1994). Short-term memory for duration in hypoxic subjects. Society for Neuroscience Abstracts, 20, 1075.

HOPKINS, R. O., \& KESNER, R. P. (1995). Item and order recognition memory in subjects with hypoxic brain injury. Brain \& Cognition, 27, 180-201.

Hopkins, R. O., Kesner, R. P., \& Goldstein, M. (1995a). Item and order recognition memory for words, pictures, abstract pictures, spatial locations, and motor responses in subjects with hypoxic brain injury. Brain \& Cognition, 27, 180-201.

Hopkins, R. O., KESNER, R. P., \& GoldSTEIN, M. (1995b). Memory for novel and familiar spatial and linguistic temporal distance information in hypoxic subjects. Journal of the International Neuropsychological Society, 1, 454-468.

Horel, J. A., Pytko-Joiner, D. E., Voytko, M. L., \& Salsbury, K. (1987). The performance of visual tasks while segments of the inferotemporal cortex are suppressed by cold. Behavioral Brain Research, $23,29-42$.

Huppert, F., \& Piercey, M. (1976). Recognition memory in amnesic patients: Effects of temporal context and familiarity of material. Cortex, 4, 3-28

JACKSON, P., KESNER, R. P., \& AMANN, K. (1994). Effects of hippocampal and medial prefrontal lesions on discrimination of duration in rats. Society for Neuroscience Abstracts, 20, 1210.

Jurado, M. A., Junque, C., Pujol, J., Oliver, B., \& Vendrell, P. (1997). Impaired estimation of word occurrence frequency in frontal lobe patients. Neuropsychologia, 35, 635-641.

KaMETANI, H., \& KeSNER, R. P. (1989). Retrospective and prospective coding of information: Dissociation of parietal cortex and hippocampal formation. Behavioral Neuroscience, 103, 84-89.

KESNER, R. P. (1989). Retrospective and prospective coding of information: Role of the medial prefrontal cortex. Journal of Experimental Brain Research, 74, 163-167.

KESNER, R. P. (1990a). Learning and memory in rats with an emphasis on the role of the hippocampal formation. In R. P. Kesner \& D. S. Olton (Eds.), Neurobiology of comparative cognition (pp. 179-204) Hillsdale, NJ: Erlbaum.

KESNER, R. P. (1990b). Memory for frequency in rats: Role of the hippocampus and medial prefrontal cortex. Behavioral \& Neural Biology. 53, $402-410$. 
KeSNER, R. P. (1998). Neurobiological views of memory. In J. L. Martinez \& R. P. Kesner (Eds.), The neurobiology of learning and memory (pp. 361-416) New York: Academic Press.

KESNER, R. P., Bolland, B. L., \& DAKIS, M. (1993). Memory for spatial locations, motor responses, and objects: Triple dissociation among the hippocampus, caudate nucleus, and extrastriate visual cortex. $E x$ perimental Brain Research, 93, 462-470.

KeSNER, R. P., \& DiMatTIA, B. V. (1987). Neurobiology of an attribute model of memory (pp. 207-277). Progress in psychobiology and physiological psychology. New York: Academic Press.

KESNER, R. P., \& HolbrooK, T. (1987). Dissociation of item and order spatial memory in rats following medial prefrontal cortex lesions. Neuropsychologia, 25, 653-664.

Kesner, R. P., Hopkins, R. O., \& Fineman, B. (1994). Item and order dissociation in humans with prefrontal cortex damage. Neuropsychologia, 32, 881-891.

KeSNER, R. P., \& NovaK, J. (1982). Serial position curve in rats: Role of the dorsal hippocampus. Science, 218, 173-174.

KeSNER, R. P., \& WILBURN, M. W. (1974). A review of electrical stimulation of the brain in context of learning and retention. Behavioral Biology, 10, 259-293.

KeSNER, R. P., \& Williams, J. M. (1995). Memory for magnitude of reinforcement: Dissociation between the amygdala and hippocampus Neurobiology of Learning \& Memory, 64, 237-244.

Kim, J. J., ClaRK, R. E., \& THompson, R. F. (1995). Hippocampectomy impairs the memory of recently, but not remotely, acquired trace eyeblink conditioned responses. Behavioral Neuroscience, 109, 195-203.

KIM, J. J., \& FANSELOW, M. S. (1992). Modality-specific retrograde amnesia of fear. Science, 256, 675-677.

Kojima, S., Matsumura, M., \& Kubota, K. (1981). Prefrontal neuron activity during delayed-response performance without imperative GO signals in the monkey. Experimental Neurology, 74, 396-407.

KoLB, B. (1974). Social behavior of rats with chronic prefrontal lesions. Physiological Psychology, 87, 466-474.

KolB, B., \& MiLner, B. (1981). Performance of complex arm and facial movements after focal brain lesions. Neuropsychologia, 19, 491-504.

LAROCHE, S., JAY, T. M., \& THIERRY, A. M. (1990). Long-term potentiation in the prefrontal cortex following stimulation of the hippocampal CAl/subicular region. Neuroscience Letters, 114, 184-190.

LEONARD, G., \& MiLnER, B. (1991). Contribution of the right frontal lobe to the encoding and recall of kinesthetic distance information. Neuropsychologia, 29, 47-58.

LEWINSOHN, P. M., Zieler, J. L., Libet, J., EyeberG, S., \& Nielson, G. (1972). Short-term memory: A comparison between frontal and nonfrontal right- and left-hemisphere brain-damaged patients. Journal of Comparative \& Physiological Psychology, 81, 248-255.

MADIGAN, S. A. (1969). Intraserial repetition and coding processes in free recall. Journal of Verbal Learning \& Verbal Behavior, 8, 828-835.

Maren, S., Aharonov, G., \& Fanselow, M. S. (1996). Excitotoxic dorsal hippocampus lesions and Pavlovian fear conditioning in rats. Society for Neuroscience Abstracts, 22, 1379.

MaRKowITSCH, H. J. (1995). Which brain regions are critically involved in the retrieval of old episodic memory? Brain Research Reviews, 21, $117-127$.

McDonald, R. J., \& White, N. M. (1993). A triple dissociation of systems: Hippocampus, amygdala, and dorsal striatum. Behavioral Neuroscience, 107, 3-22.

Meck, W. H., Church, R. M., \& Olton, D. S. (1984). Hippocampus, time and memory. Behavioral Neuroscience, 98, 3-22.

MiLnER, B. (1964). Some effects of frontal lobectomy in man. In J. M. Warren \& K. Akert (Eds.), The frontal granular cortex and behavior (pp. 313-334). New York: McGraw-Hill.

MILNER, B. (1971). Interhemispheric differences in the localization of psychological processes in man. British Medical Bulletin, 27, 272-277.

Milner, B., Petrides, M., \& SMith, M. L. (1985). Frontal lobes and the temporal organization of memory. Human Neurobiology, 4, 137-142.

Morris, R. G., Ahmed, S., Syed, G. M., \& ToONE, B. K. (1993). Neural correlates of planning ability: Frontal lobe activation during the Tower of London test. Neuropsychologia, 31, 1367-1378.
Moyer, J. R., JR., Deyo, R. A., \& Disterhoft, J. F. (1990). Hippocampectomy disrupts trace eye-blink conditioning in rabbits. Behavioral Neuroscience, 104, 243-252.

Mumby, D. G., Wood, E. R., \& PinEL, J. P. J. (1992). Object-recognition memory is only mildly impaired in rats with lesions of the hippocampus and amygdala. Psychobiology, 20, 18-27.

Nadel, L., \& Moscovitch, M. (1997). Memory consolidation, retrograde amnesia and the hippocampal complex. Current Opinion in Neurobiology, 7, 217-227.

NIKI, H. (1974a). Prefrontal unit activity during delayed alternation in the monkey. I. Relation to direction of response. Brain Research, $\mathbf{6 8}$, $185-196$

NIKI, H. (1974b). Prefrontal unit activity during delayed alternation in the monkey. II. Relation to absolute versus relative direction of response. Brain Research, 68, 197-204.

O'KeEfe, J., \& NADEL, L. (1978). The hippocampus as a cognitive map. Oxford: Clarendon.

OLton, D. S. (1983). Memory functions and the hippocampus. In W. Seifert (Ed.), Neurobiology of the hippocampus (pp. 335-373). New York: Academic Press.

OLTON, D. S. (1986). Hippocampal function and memory for temporal context. In R. L. Isaacson \& K. H. Pribram (Eds.), The hippocampus (Vol. 3, pp. 281-298). New York: Plenum.

Otron, D. S., Wenk, G. L., Church, R. M., \& Meck, W. H. (1988). Attention and the frontal cortex as examined by simultaneous temporal processing. Neuropsychologia, 26, 307-318.

Otto, T., \& Eichenbaum, H. (1992). Complementary roles of the orbital prefrontal cortex and the perirhinal-entorhinal cortices in an odor-guided delayed-nonmatching-to-sample task. Behavioral Neuroscience, 106, 762-775

Owen, A. M., Downes, J. J., Sahakian, B. J., Polkey, C. E., \& RobBINS, T. W. (1990). Planning and spatial working memory following frontal lobe lesions in man. Neuropsychologia, 28, 1021-1034.

Passingham, R. (1978). Information about movements in monkeys (Macaca mulatta) with lesions of dorsal prefrontal cortex. Brain Research, 152, 313-328.

Petrides, M., \& Milner, B. (1982). Deficits on subject-ordered task after frontal- and temporal-lobe lesions in man. Neuropsychologia, 20, 249-262.

Pribram, K. H., \& TubBs, W. E. (1967). Short-term memory, parsing. and the primate frontal cortex. Science, 156, 1765-1767.

Ragozzino, M. E., \& Kesner, R. P. (1996). Learning and memory for taste information: Role of the agranular insular cortex. Society for Neuroscience Abstracts, 22, 1868.

Sagar, H. J., Gabrieli, J. D. E., Sullivan, E. V., \& Corkin, S. (1990). Recency and frequency discrimination in the amnesic patient H.M. Brain, 113, 581-602

Santi, A., Weise, L., \& Kuiper, D. (1995). Memory for event duration in rats. Learning \& Motivation, 26, 83-100.

SCHACTER, D. L. (1987). Implicit memory: History and current status. Journal of Experimental Psychology: Learning, Memory, \& Cognition, 13, $501-518$

SCHACTER, D. L., \& Tulving, E. (1994). Memory systems 1994. Cambridge, MA: MIT Press.

Shallice, T. (1982). Specific impairments of planning. Philosophical Transactions of the Royal Society of London: Series B, 298, 199-209.

SLOTNICK, B. M. (1967). Disturbances of maternal behavior in the rat following lesions of the cingulate cortex. Behavior, 29, 204-236.

SQuiRe, L. R. (1994). Declarative and nondeclarative memory: Multiple brain systems supporting learning and memory. In D. L. Schacter \& E. Tulving (Eds.), Memory svstems 1994 (pp. 203-231). Cambridge, MA: MIT Press

Squire, L. R., Nadel, L., \& Slater, P. (1981). Anterograde amnesia and memory for temporal order. Neuropsychologia, 19, 141-146.

Stamm, J. S. (1955). The function of the median cerebral cortex in maternal behavior of rats. Journal of Comparative \& Physiological Psychology, 48, 347-356.

SWANSON, L. W. (1981). A direct projection from Ammon's horn to prefrontal cortex in the rat. Brain Research, 217, 150-154. 
Tulving, E. (1983). Elements of episodic memory. Oxford: Clarendon.

Uylings, H. B. M., \& van Eden, C. G. (1990). Qualitative and quantitative comparison of the prefrontal cortex in rat and in primates, including humans. In H. B. M. Uylings, C. G. Van Eden, J. P. C. De Bruin, M. A. Corner, \& M. G. P. Feenstra (Eds.), Progress in brain research (Vol. 85, pp. 31-62). Amsterdam: Elsevier.

Weisend, M. P., Astur, R. S., \& SUTHERLAND, R. J. (1996). The specificity and temporal characteristics of retrograde amnesia after hippocampal lesions. Society for Neuroscience Abstracts, 22, 1118.
Weiskrantz, L., \& SaUnders, C. (1984). Impairments of visual object transforms in monkeys. Brain, 107, 1033-1072.

Witter, M. P., Groenewegen, H. J., Lopes Da Silva, F. H., \& LohMAN, A. H. M. (1989). Functional organization of the extrinsic and intrinsic circuitry of the parahippocampal region. In G. A. Kerkut \& J. W. Phillis (Eds.), Progress in neurobiology (Vol. 33, pp. 161-253). Oxford: Pergamon.

(Manuscript received July 11, 1997; revision accepted for publication February 12, 1998.) 